\title{
Retorno ao trabalho? Indicadores de saúde mental em professores durante a pandemia da COVID-19
}

\author{
Roberto Moraes Cruz ${ }^{1}$ \\ https://orcid.org/0000-0003-4671-3498 \\ Ricelli Endrigo Ruppel da Rocha ${ }^{2}$ \\ https://orcid.org/0000-0002-4277-1407 \\ Solange Andreoni ${ }^{3}$ \\ https://orcid.org/0000-0003-4589-9876 \\ Andrea Duarte Pesca ${ }^{4}$ \\ https://orcid.org/0000-0002-5471-3810
}

\section{Resumo}

O objetivo deste estudo foi rastrear indicadores de saúde mental dos docentes de uma instituição de educação infanto-juvenil da regiâo sul do Brasil. Trata-se de um estudo descritivo com 84 docentes $(80,7 \%$ do total), que responderam a um formulário via web, composto por três instrumentos: a) questionário demográfico e sócio-ocupacional; b) a escala Dass-21; c) escala de sintomas de saúde mental relacionados ao trabalho (IP-T). Os resultados mostraram que $82,1 \%$ dos participantes estavam preocupados com a exposição ao novo coronavírus, $6,0 \%$ referiram não estar em isolamento social, $84,5 \%$ indicam ter conhecimentos sobre a pandemia e $85,7 \%$ apresentam baixa expectativa de retorno ao ambiente de trabalho. A ansiedade $(21,7 \%$ e $27,6 \%$ ) e a depressão $(28,9 \%$ e $28,5 \%$ ), tanto na escala Dass-21 quanto na escala IP-T, são as alteraçóes mais frequentes na saúde mental dos docentes. Nos resultados das associaçóes, os docentes do sexo feminino, com faixa etária de 46 à 56 anos e solteiros, apresentaram mais chances para desenvolver ansiedade e depressáo $(p<0,05)$. Por outro lado, conviver com pessoas

${ }^{1}$ Professor Doutor do Programa de Pós-Graduaçáo em Psicologia da Universidade Federal de Santa Catarina (UFSC), Florianópolis, SC, Brasil. E-mail: robertocruzdr@gmail.com.

${ }_{2}$ Professor Doutor do Programa de Mestrado Profissional em Educação Básica e Programa de Mestrado Acadêmico em Desenvolvimento e Sociedade da Universidade Alto Vale do Rio do Peixe (UNIARP), Caçador, Brasil. E-mail: ricellie@uniarp.edu.br.

${ }^{3}$ Professora Doutora do Programa de Pós-Graduação em Saúde Coletiva da Universidade Federal de São Paulo (UNIFESP), São Paulo, Brasil.

${ }^{4}$ Professora Doutora da Faculdade de Motricidade Humana da Universidade de Lisboa (ULISBOA), Lisboa, Portugal. E-mail: adpesca07@gmail.com. 
consideradas em situação de risco para a COVID-19 reduzem as chances $(0,91)$ de manifestação de sintomas de ansiedade e depressão $(\mathrm{p}<0,05)$. Em resumo, os indicadores de saúde mental dos professores em meio à pandemia da COVID-19 devem ser rastreados para promover o retorno ao trabalho de forma segura e responsável.

Palavras-chave: Retorno ao trabalho, Professor, Saúde mental, COVID-19, Pandemia.

\section{Returning to work? Mental health indicators of the teachers during the COVID-19 pandemic}

\section{Abstract}

The aim of this study was to track the mental health indicators of the teachers in a given institution of children and youth education in the south region of Brazil. It is a descriptive study with 84 teachers ( $80.7 \%$ total), who answered a form via web, made up of three tools: a) social occupational demographic questionnaire; b) the Dass-21 scale; c) mental health symptoms scale related to work (IP-T). The results showed that $82.1 \%$ of the participants were worried with the exposure to the new coronavirus, $6.0 \%$ informed not being under social distancing, $84.5 \%$ showed being aware of the pandemic and $85.7 \%$ showed low expectations of returning to work environment. Anxiety $(21.7 \%$ and $27.6 \%)$ and depression $(28.9 \%$ and $28.5 \%$ ), both in the Dass-21 and IP-T scale, are the more frequent mental health alterations in teachers. In the association results, the female teachers within the 46 to 56 age group and single, showed more chances to develop anxiety and depression $(p<0.05)$. On the other hand, interacting with people who are under risk situation to catch COVID-19 reduces the chances to manifest anxiety and depression symptoms $(\mathrm{p}<0.05)$. In summary, the mental health indicators of the teachers during the COVID-19 pandemic should be tracked to promote the returning to work in a responsible and safe way.

Keywords: Return to work, Mental health, COVID-19, Pandemics.

\section{Introdução}

A pandemia da COVID-19 produziu impactos severos na saúde pública, assim como nas condiçóes de vida das pessoas na maior parte dos países. Enquanto a pandemia segue o seu curso, há a necessidade das diferentes instâncias do governo, entidades da sociedade civil e profissionais de diferentes ocupaçóes em prover assistência à saúde, segurança e recursos necessários à redução da velocidade de difusão da doença e na mitigação de seus resultados na saúde das pessoas (TAN et al., 2020; THE LANCET, 2020).

As medidas de isolamento social e de restrição da mobilidade promoveram a aceleração de mudanças nas rotinas de trabalho por meio da informatização e digitalização de processos e procedimentos e no incremento da prestação de serviços on-line, especialmente no campo da educação, nas 
atividades administrativas, logística e vendas (AQUINO et al., 2020; IIVARI et al., 2020). O teletrabalho e todas as variaçôes de teleatendimento, em diferentes ramos da economia foram intensificados com a pandemia da COVID-19. O uso de tecnologias e meios de comunicação virtuais alcançaram níveis nunca antes experimentados, repercutindo na qualidade e na disponibilidade de serviços de internet (MELLO; TER-MINASSIAN, 2020).

Em meio a essa dinâmica social, foram implementadas medidas que interromperam completamente ou limitaram fortemente a reunião de pessoas em locais públicos e o funcionamento de entidades educacionais. A duração dessas medidas, promovidas de forma extensiva, refletiu nas interaçóes sociais e de trabalho, na empregabilidade e sustento econômico das pessoas, assim como comprometeram as atividades de escolas, universidades e demais centros de formação profissional (BARROS-DELBEN; et al., 2020; CRUZ et al., 2020).

No setor educacional, a tentativa de promover o ensino por meio de atividades síncronas e assíncronas, a fim de mitigar os efeitos do distanciamento das atividades planejadas entre alunos e professores, revelouse uma das poucas alternativas encontradas pelas instituiçôes educativas, tendo em vista a impossibilidade de as pessoas frequentarem os ambientes com segurança e a necessidade de manter contratos de trabalho. Porém, a transição do ensino presencial para um modelo digital de educação, mesmo considerando todas as experiências educacionais nesse sentido, não é um movimento simples e de fácil absorção social (UNESCO, 2020). Os problemas estruturais de acesso à banda larga e aos meios tecnológicos de comunicaçáo por internet ainda é uma realidade desigual para um grande contingente de pessoas. Há, ainda, a falta de treinamento de professores para realizar educação digital (no setor público e privado), despreparo dos gestores de instituiçóes de ensino para planejar açóes de médio e longo prazo nessa direçáo, além das incertezas e inseguranças típicas de um processo de adaptação cultural para o qual não se sabe ao certo suas chances de sucesso.

Nesse contexto, a progressão da pandemia da COVID-19 se revelou severa em termos de propagação da infecção, atingimento da saúde física e mental das pessoas e taxas de mortalidade sem precedentes. $\mathrm{O}$ novo coronavírus atingiu pessoas de diferentes características sociodemográficas e os mais vulneráveis, em graus diferenciados de severidade, desde a 
manifestação de sintomas de gripe e outros problemas secundários até o acometimento de insuficiência respiratória aguda, que requer cuidados hospitalares intensivos, incluindo o uso de ventilação mecânica (CDC COVID-19 RESPONSE TEAM, 2020). A falta de imunização biológica ao novo coronavírus e, especialmente, a sua elevada facilidade de propagaçáo têm caracterizado a pandemia da COVID-19 como um problema de saúde pública internacional e com repercussóes ainda não conhecidas totalmente (FARO et al., 2020).

Do ponto de vista da saúde mental, verifica-se a exacerbação sintomas de transtornos de humor, especialmente ansiedade, depressão, além de episódios de pânico, estresse agudo e pós-traumático, não apenas entre os profissionais, mas na população de modo geral (TEMSAH et al., 2020; WANG et al., 2020). Pesquisas recentes indicam a necessidade de monitoramento dos impactos da pandemia da COVID-19 na saúde mental, assim como na implementação de programas controle e prevenção de crises e de quadros importantes de transtornos mentais e do comportamento, tendo em vista a duração do confinamento social, o temor da contaminação, as pressões por sustentação econômica e a necessidade de retorno ao trabalho (LEE, 2020; LIU et al., 2020; SCHMIDT et al., 2020; THE LANCET, 2020).

Para minimizar esses impactos provocados por situações de crises e pandemias, como o caso do novo coronavírus, as instituiçôes educacionais precisam adotar mecanismos adaptativos inovadores para enfrentar os desafios de realinhamento de rotinas e interaçóes no ambiente de trabalho, especialmente entre professores e alunos (BIRON et al., 2020). O retorno ao trabalho durante a pandemia COVID-19 é uma pressão social constante para muitos trabalhadores e empresas, inclusive no setor educacional. Nesse sentido, além de preparar ambiente de trabalho, ao dispor de normas de proteção e cuidado de higienização, é importante investigar o grau de vulnerabilidade dos trabalhadores em termos de saúde mental (SOMINSKY et al., 2020).

O objetivo deste estudo foi rastrear indicadores de saúde mental dos professores de uma instituição de prestação de serviços educacionais que possam auxiliar na compreensão dos efeitos do confinamento e do isolamento social na capacidade de trabalho e no processo retorno gradual ao ambiente de trabalho. 


\section{Método}

\section{Delineamento e Contexto}

Foi realizado um estudo descritivo sobre indicadores de saúde mental em professores de uma instituição de educação infanto-juvenil que atua na região sul do Brasil. Estudos descritivos têm por interesse descrever as características de determinado evento ou fenômeno em uma população e estabelecer relaçóes entre as variáveis de desfecho e as características dos participantes (RANGANATHAN; AGGARWAL, 2018).

Desde a segunda quinzena do mês de março de 2020, todos os professores da instituição encontram-se em atividades de teletrabalho. Está em fase de planejamento o retorno às dependências da instituição, o que implica na organização de medidas de higienização dos ambientes de trabalho e de sensibilização de todos os funcionários e prestadores de serviços quanto aos procedimentos de higienizaçáo dos equipamentos de trabalho, uso de máscaras e demais cuidados de proteção à contaminação pelo coronavírus.

\section{Participantes}

A instituição conta com quadro geral de 145 funcionários (entre professores e demais funcionários das atividades administrativas/operacionais), distribuídos em 4 cidades da regiáo Sul do Brasil. De um total de 104 professores ativos da instituição, 84 deles responderam efetivamente à pesquisa $(80,7 \%)$, resultando em uma amostra de conveniência. Nesta pesquisa foram considerados os resultados coletados apenas com a população-alvo: professores.

\section{Instrumentos de coleta de dados}

Foi disponibilizado aos participantes um formulário único, organizado em uma plataforma para respostas via web, contendo os seguintes instrumentos de coleta de dados:

a) Questionário sobre o perfil dos participantes, visando caracterizar aspectos demográficos (sexo, faixa etária, estado civil e tipo de atividade) e 
sócio-ocupacionais (contato com pessoas diagnosticadas com o novo coronavírus, grau de preocupaçáo com a pandemia, conhecimentos acerca da pandemia, grau de isolamento social e se convivem atualmente com pessoas do chamado grupo de risco - idosos, pessoas com doenças crônicas, gestantes e bebês, assim como características da atividade de ensino, carga horária semanal de docência e expectativa de retorno ao ambiente de trabalho.

b) Escala de autorrelato DASS-21, em sua versão reduzida, adaptada e validada para o português do Brasil, indicada para rastrear estados negativos. A escala é composta por 21 itens divididos em três subescalas: depressão, ansiedade e estresse (MARTINS et al., 2019) e, para tal, utiliza 3 níveis de mensuração para cada item investigado: de 0 (zero) "não se aplica de maneira nenhuma" a 3 (três) "aplica-se muito, ou na maioria do tempo", com base na instrução "em que medida cada afirmação se aplica a você no curso da última semana"

c) Inventário de saúde mental relacionada ao trabalho - IP-T (GUILLAND et al., 2018), composto por 50 itens e 5 subescalas: ansiedade, depressão e transtornos somatoformes, habilidades sociais e bem-estar no trabalho. O IP-T foi escolhido em função da possibilidade de comparação com duas subescalas da Dass-21 (ansiedade e depressão), além de rastrear repertórios positivos de enfrentamento de sintomas, relevantes ao contexto de investigação (subescalas habilidades sociais e bem-estar no trabalho) e, ainda, pelo fato do IP-T ter validado em amostras de trabalhadores brasileiros. Baseado na instrução "Com que frequência esses comportamentos ocorreram nas últimas 4 semanas?” é oferecida uma escala de resposta - nunca, às vezes, geralmente, sempre.

\section{Procedimentos}

A sensibilização para a coleta de dados foi realizada por meio de uma comunicação interna promovida pela administração central da instituição e dirigida a todos os funcionários da Instituição via e-mail. Nessa comunicação destacava-se a importância da participação de todos na pesquisa sobre "Saúde mental e retorno ao trabalho", tendo em vista o planejamento de retorno gradual dos funcionários ambiente de trabalho a partir do mês de setembro de 2020. A final dessa comunicação, havia uma breve explicação da pesquisa, seu 
objetivo e possibilidade contribuição ao planejamento do trabalho, assim como foi disponibilizado um acesso por hiperlink ao formulário de pesquisa.

O acesso inicial ao formulário foi precedido de uma autorização expressa por parte do participante em concordar em responder aos instrumentos de pesquisa, com base em um Termo de Consentimento Livre e Esclarecido, sem a qual não era possível responder às informaçôes solicitadas. A coleta de dados foi realizada integralmente no mês de julho. Para todos os participantes foi facultada a possibilidade de acesso a um relatório completo dos dados pesquisados, evidentemente com a não identificaçáo dos participantes, assim como um feedback personalizado dos resultados da pesquisa, agendada previamente com os pesquisadores por e-mail ou whatsapp.

\section{Tratamento e Análise de Dados}

Os dados foram organizados em uma planilha Excel e analisados por meio do programa Statistical Package for the Social Sciences (SPSS, versão 22). As respostas foram analisadas integralmente por meio de estatísticas descritivas (percentual de ocorrência, média e desvio-padrão) para descrever o perfil demográfico e sócio-ocupacional dos participantes. Foram realizadas correlaçóes entre os indicadores de sintomas de agravos à saúde mental aferidos pelos instrumentos escalares e realizada regressão logística para explorar os preditores do perfil dos participantes frente aos indicadores de saúde mental.

\section{Resultados}

\section{Perfil dos Participantes}

O mapeamento do perfil demográfico dos participantes da pesquisa (Tabela 1) revelou a predominância do sexo feminino $(75,0 \%)$ e do estado civil casado/união estável (61,9\%). A média de idade foi de 31,4 anos, com faixa etária entre 24 e $34(38,1 \%)$, e os que referiram ter entre 1 e 2 filhos $(48,8 \%)$. 
Tabela 1 - Perfil demográfico dos participantes (N=84)

\begin{tabular}{llll}
\hline Variáveis & Categoria & $\mathrm{N}$ & $\%$ \\
\hline Sexo & Feminino & 63 & 75,0 \\
Faixa Etária & Masculino & 21 & 25,0 \\
& $24-34$ & 32 & 38,1 \\
& $35-45$ & 28 & 33,3 \\
& $46-56$ & 16 & 19,0 \\
\multirow{3}{*}{ Estado Civil } & Acima de 56 & 32 & 38,1 \\
& Casado(a)/Uniāo estável & 52 & 61,9 \\
& Separado(a)/Divorciado(a) & 12 & 14,3 \\
\multirow{3}{*}{ Filhos } & Solteiro(a) & 17 & 20,2 \\
& Viúvo(a) & 3 & 3,6 \\
& Náo & 35 & 41,7 \\
& $1-2$ & 41 & 48,8 \\
\hline
\end{tabular}

Fonte: Autores (2020).

O perfil sócio-ocupacional (Tabela 2) retrata participantes preocupados com a exposição ao novo coronavírus (82,1\%), que residem com pessoas do grupo de risco para a doença causadora da pandemia - idosos, pessoas com doenças crônicas, gestantes e bebês $(64,3 \%)$, embora a maior parte não tenha tido contado até o momento com pessoas que foram diagnosticadas com COVID-19 (84,5\%). Do total, apenas 6,0\% referiram não estar em isolamento social total ou parcial, sendo que $84,5 \%$ indicam ter conhecimentos sobre a pandemia para prover cuidados e tomar decisóes. Os docentes atuam majoritariamente realizando atividade síncronas e assíncronas com estudantes $(91,7 \%)$ e referem baixa expectativa, no momento, de retorno ao ambiente de trabalho $(85,7 \%)$. A carga horária de trabalho está dimensionada atualmente para a realizaçáo de atividades de videoconferência, preparação de atividades de ensino e sistema de avaliação, eventualmente ultrapassando as 20 horas semanais para $39,3 \%$ dos participantes. 
Tabela 2 - Perfil sócio-ocupacional dos participantes ( $\mathrm{N}=84)$

\begin{tabular}{llll}
\hline Variáveis & Categoria & $\mathrm{N}$ & $\%$ \\
\hline Preocupaçáo com a exposiçáo & Sim & 69 & 82,1 \\
& Não & 15 & 17,9 \\
Contato com pessoas/Covid-19 & Sim & 13 & 15,5 \\
& Não & 71 & 84,5 \\
Convivência com grupo de risco & Sim & 54 & 64,3 \\
& Não & 30 & 35,7 \\
Isolamento social & Total & 44 & 52,4 \\
& Parcial & 35 & 41,7 \\
Conhecimento sobre a COVID-19 & Náo & 5 & 6,0 \\
& Suficiente & 71 & 84,5 \\
Atividade de ensino & Insuficiente & 13 & 15,5 \\
& Síncronas e Assíncronas & 77 & 91,7 \\
Carga horária semanal & Somente Assíncronas & 7 & 8,30 \\
\multirow{2}{*}{ Expectativa de retorno AT } & Até 20h & 52 & 61,9 \\
& Acima de 20h & 33 & 39,3 \\
& Baixa & 72 & 85,7 \\
& Alta & 12 & 14,3 \\
\hline
\end{tabular}

Fonte: Os autores (2020).

\section{Sintomas de Agravos à Saúde Mental}

O rastreamento de alteraçóes na saúde mental realizados por meio das escalas Dass-21 e IPT identificou, respectivamente e de forma comparativa os sintomas percentualmente mais frequentes: ansiedade (21,7\% e 27,6\%), depressão $(28,9 \%$ e $28,5 \%)$ e (Tabela 2$)$.

Tabela 3 - Percentual de sintomas de agravos à saúde mental mais e menos frequentes baseados no Dass-21 e IPT $(\mathrm{N}=85)$

\begin{tabular}{lllll}
\hline \multirow{2}{*}{ Sintomas } & \multicolumn{3}{c}{ Dass-21 } & \multicolumn{2}{c}{ IPT } \\
\cline { 2 - 5 } & $\begin{array}{l}\text { Menos } \\
\text { frequentes } \\
(\%)\end{array}$ & $\begin{array}{l}\text { Mais } \\
\text { frequentes } \\
(\%)\end{array}$ & $\begin{array}{l}\text { Menos } \\
\text { frequentes (\%) }\end{array}$ & $\begin{array}{l}\text { Mais } \\
\text { frequentes } \\
(\%)\end{array}$ \\
\hline Ansiedade & 78,3 & 21,7 & 72,4 & 27,6 \\
Depressáo & 71,1 & 28,9 & 71,5 & 28,5 \\
Estresse & 87,3 & 12,7 & - & - \\
Transtornos Somatoformes & - & - & 66,6 & 33,4 \\
\hline
\end{tabular}

Fonte: Autores (2020).

Foram verificadas as validades convergente e discriminante das subescalas intrínsecas ao Dass-21 e ao IPT e entre as escalas. A Tabela 4 resume as correlaçóes obtidas, demonstrando valores moderados a altos entre as subescalas. As correlaçóes entre ansiedade e depressão nas escalas Dass-21 e 
IP-T foram, respectivamente, 0,84 e 0,79 , sendo considerados significativos para procedimentos de rastreio comparados.

Tabela 4 - Matriz de correlação entre os escores das escalas Dass-21 e IP-T.

\begin{tabular}{|c|c|c|c|c|c|c|c|c|}
\hline \multirow{2}{*}{ Escalas } & \multicolumn{3}{|c|}{ Dass-21 } & \multicolumn{5}{|c|}{ IPT } \\
\hline & Ans. & Dep. & Estr. & Ans. & Dep. & TS. & HS & BET \\
\hline Dass-21 Ansiedade & 1 & & & & & & & \\
\hline Dass-21 Depressão & 0,80 & 1 & & & & & & \\
\hline Dass-21 Estresse & 0,73 & 0,78 & 1 & & & & & \\
\hline IPT Ansiedade & 0,82 & 0,65 & 0,52 & 1 & & & & \\
\hline IPT Depressáo & 0,69 & 0,79 & 0,44 & 0,59 & 1 & & & \\
\hline IPT Transt. Somat. & 0,65 & 0,43 & 0,41 & 0,72 & 0,54 & 1 & & \\
\hline IPT Hab. Sociais & $-0,21$ & $-0,19$ & $-0,23$ & 0,28 & 0,74 & 0,69 & 1 & \\
\hline IPT B-E no Trab. & $-0,23$ & $-0,19$ & $-0,21$ & 0,72 & 0,69 & 0,70 & 0,81 & 1 \\
\hline
\end{tabular}

Fonte: Autores (2020).

As subescalas habilidades sociais e de bem-estar no trabalho da IPT apresentaram correlaçóes negativas com os sintomas de ansiedade, depressão e estresse da escala Dass-21, confirmando a expectativa de que ambas sinalizam a manutenção de um certo potencial de enfrentamento dos sintomas de agravos à saúde mental. A subescala transtorno somatoforme (IP-T) mostrou maior correlação com a subescala de ansiedade da escala Dass-21. A subescala transtornos somatoformes da IPT apresentou correlaçáo importante $(0,65)$ com a subescala ansiedade, indicando que variáveis fisiológicas, tais como problemas com o sono, palpitaçóes, dores no corpo, aumento da frequência cardíaca e parestesias mostram-se associadas a preocupaçóes com a saúde em geral e medo da contaminação.

\section{Correlaçáo entre os Indicadores de Saúde Mental e o Perfil dos Professores}

Para ampliar o grau de compreensão dos resultados, foi realizada uma regressão logística para medir os efeitos de variáveis do perfil demográfico e sócio-ocupacional dos professores com a probabilidade de manifestação de sintomas de agravos à saúde mental. A regressão logística permitiu identificar a razão de chances de determinadas variáveis do perfil dos professores significativamente associadas a frequência de sintomas de ansiedade e depressão, considerando os escores acima dos pontos de corte das escalas de rastreio utilizadas comparativamente. Os resultados apresentados na Tabela 5 
mostra a razão de chances associada ao respectivo intervalo de confiança e à significância (valor de p) da variável explicada.

Tabela 5 - Razão de chances da ansiedade e depressão conforme o perfil dos participantes $(\mathrm{N}=84)$

\begin{tabular}{lcccc}
\multicolumn{1}{c}{ Variáveis } & $\begin{array}{c}\text { Razáo de } \\
\text { chance }\end{array}$ & Inf. & Sup. & $\begin{array}{c}\text { Intervalo } \\
p\end{array}$ \\
\hline Sexo (fem) & 1,62 & 1,143 & 4,342 & $0,04^{*}$ \\
Faixa etária (46-56 anos) & 1,41 & 1,077 & 3,451 & $0,03^{*}$ \\
Estado civil (solteiro) & 1,88 & 1,212 & 5,312 & $0,03^{*}$ \\
Preocupaçáo com a COVID-19 (Sim) & 1,56 & 0,634 & 3,323 & 0,06 \\
Convivência com grupo de risco (Sim) & 0,91 & 0,879 & 5,442 & $0,04^{*}$ \\
Carga horária (acima de 20h) & 1,23 & 0,953 & 3,523 & 0,63 \\
\hline
\end{tabular}

Fonte: Autores (2020). Nota. *valor de $p<0,05$.

As mulheres apresentaram 1,62 vezes mais chances do que os homens de apresentarem sintomas de ansiedade e depressão, assim como os participantes da faixa etária de 46-56 anos $(1,41)$ e os solteiros $(1,88)$, sendo todos os valores significativos $(p<0,05)$. Referir preocupação com a pandemia da COVID-19 aumenta em 1,56 vezes as chances dos participantes em manifestarem sintomas ansiedade e depressão, assim como trabalhar com carga horária semanal acima de 40 horas, embora sejam valores que não alcançaram significância estatística. Por outro lado, conviver com pessoas consideradas em situação de risco para a COVID-19 reduzem as chances $(0,91)$ de manifestação de sintomas de ansiedade e depressão, o que pode ser considerado um fator protetivo à agravos a saúde mental, provavelmente em função do aumento nos cuidados (auto)implementados.

\section{Discussáo}

A expansão da pandemia do novo coronavírus revelou um aumento de transtornos mentais comuns, especialmente transtornos adaptativos, que 
englobam alteraçóes emocionais e comportamentais debilitantes, associados a eventos estressantes, episódios de pânico, ansiedade e depressão (SCHWARTZ et al., 2020). Pessoas tendem a se sentir ansiosas e inseguras quando o ambiente muda ou sob pressóes constantes. No caso de surtos de doenças infecciosas, quando a causa ou progressão da doença e os resultados não são claros, crescem os rumores e ocorrem atitudes de mente fechada (LIANG et al., 2020). Observa-se que os problemas de saúde mental, especialmente os níveis de ansiedade e estresse, aumentaram significativamente desde o aparecimento da pandemia do coronavírus e seus impactos socioeconômicos e nas restriçóes de mobilidade humana (USHER; DURKIN; BHULLAR, 2020).

Medo, preocupação e estresse são respostas adaptativas a ameaças percebidas ou reais, embora, às vezes, possam estar exacerbadas no contexto em que ocorrem (WORLD HEALTH ORGANIZATION, 2020). Além do temor de ser infectado pelo coronavírus, os esforços por corresponder às exigências provocadas pelas mudanças de rotina nos âmbitos familiar, social e de trabalho aceleraram a manifestação de alteraçóes de humor e de afeto nas pessoas, especialmente aquelas com histórico de doenças ou que pertencem aos grupos de risco à doença (BARROS-DELBEN et al., 2020). Diante desse cenário, é importante considerar os impactos na saúde mental nos professores, considerando a atual experiência de confinamento e isolamento social, assim como sua relação com as expectativas de retorno ao ambiente de trabalho e à interação com os alunos.

Alteraçóes importantes na saúde mental têm impacto no funcionamento em tarefas habituais (domésticas ou de trabalho), geralmente associados à desmotivação, desatenção, desconcentração, anedonia (perda de prazer em fazer as coisas), aumento de erros e pequenos acidentes, propensão ao afastamento do trabalho, redução do interesse na interação com as outras pessoas. A presença de transtornos somatoformes entre os participantes $(13,6 \%)$ e os níveis de ansiedade acentuados mostrado em ambas as escalas de rastreio (16,2 e 25,5\%), mostram que as preocupaçóes e temores frente à situação da pandemia estão associadas aos desconfortos físicos e fisiológicos, o que contribui para o incremento da ansiedade.

Nesta pesquisa, as mulheres apresentaram maior probabilidade de manifestação de sintomas de agravos à saúde mental do que os homens. Agravos à saúde mental com maior ênfase nas mulheres também foi 
identificado em outros estudos, que referem níveis mais elevados de ansiedade (WANG et al., 2020), estresse, depressão (WORLD HEALTH ORGANIZATION, 2020) e de sofrimento psicológico (QIU et al., 2020). Participantes com ensino superior também apresentaram maior frequência de sintomas de agravos à saúde mental em comparação com os que possuem ensino médio. Isso corrobora os achados Qiu et al. (2020) em relação à angústia, que apontam que pessoas com ensino superior tendem a desenvolver mais esse sintoma, que possivelmente está associado com a autoconsciência da própria saúde e maior acesso à informação, diferentemente de pessoas com menor nível de escolaridade, conforme entende Lei et al. (2020).

Professores solteiros apresentaram maiores chances de manifestarem sintomas de ansiedade e depressão. Shacham et al. (2020) observa que manter níveis de satisfação com a elevados e relacionamentos afetivos são fatores protetivos para a reduçáo do sofrimento psicológico. Um estudo realizado em um hospital, os médicos - que atuam na atividade fim - apresentaram comprometimento na saúde mental maiores quando comparado com funcionários que atuam nos setores administrativos - atividade meio, demonstrando resultados alinhados com os achados deste estudo (LU et al., 2020).

Pertencer à faixa etária acima dos 50 mostrou ter menor chance se apresentar sintomas de agravos à saúde mental frente às demais faixas etárias. Os achados de Wang et al. (2020) e de Qiu et al. (2020) indicaram, de forma diferente, que pessoas com mais de 40 anos tendem a apresentar maior risco de ansiedade e que pessoas com mais de 60 anos tendem a apresentar maior sofrimento psicológico. Hipotetiza-se, neste estudo, que o grupo de maior faixa etária tem provavelmente têm aumentado os cuidados de exposição social e que, os grupos de menor faixa etária tendem a apresentar maior mobilidade social e, portanto, mais exposição à COVID-19.

Outro fator de risco para agravos à saúde mental constatado foi a preocupação com a pandemia da COVID-19. O medo e as percepçóes distorcidas do risco são apontados por Shigemura et al. (2020) como possíveis motivadores de comportamentos sociais negativos, que podem acarretar outros sintomas como angústia, comportamentos de risco e distúrbios relacionados à saúde - em que se inclui ansiedade, depressão, somatização e transtorno de estresse pós-traumático -, assim como a diminuição da percepção de saúde (ASMUNDSON; TAYLOR, 2020). 


\section{Conclusáo}

O objetivo principal deste estudo foi rastrear indicadores de saúde mental de professores de uma instituição educacional infanto-juvenil da região Sul do Brasil. Os principais achados mostraram que os docentes estáo preocupados com a exposição ao novo coronavírus e a maioria está em isolamento social e com baixa expectativa de retorno ao trabalho. Além disso, as alteraçóes na saúde mental mais frequentes nos docentes foram a ansiedade e a depressão, com maiores chances de desenvolver estes problemas os docentes do sexo feminino, com faixa etária de 46 à 56 anos e solteiros. Por outro lado, conviver com pessoas consideradas em situação de risco para a COVID-19 reduzem as chances de manifestação de sintomas de ansiedade e depressão.

Avalia-se que os resultados encontrados possam auxiliar no rastreio de indicadores de saúde mental dos professores em meio à pandemia da COVID19, tendo em vista as necessidades de promover o retorno ao trabalho de forma segura e responsável. Nâo há, contudo, expectativas de generalização dos achados desta pesquisa para outras instituiçôes similares.

\section{Referências}

AQUINO, E. M. L. et al. Medidas de distanciamento social no controle da pandemia de COVID-19: potenciais impactos e desafios no Brasil. Ciência \& Saúde Coletiva, v. 25, p. 2423-2446, 2020. Disponível em: http://www.scielo.br/scielo.php?script=sci_arttext\&pid=S141381232020006702423\&nrm=iso. Acesso em: 2 jul. 2020.

APÓSTOLO, J.; TANNER, B.; ARFKEN, C. Análise fatorial confirmatória da versão portuguesa da Depression Anxiety Stress Scale-21. Revista LatinoAmericana de Enfermagem, v. 20, n. 3, p. 590-596, 2012. Disponível em: http://www.periodicos.usp.br/rlae/article/view/48583. Acesso em: 1 jul. 2020 .

ASMUNDSON, G. J. G.; TAYLOR, S. How health anxiety influences responses to viral outbreaks like COVID-19: What all decision-makers, health authorities, and health care professionals need to know. Journal of 
Anxiety Disorders, v. 71, p. 102211, 2020. Disponível em: https://pubmed.ncbi.nlm.nih.gov/32179380. Acesso em: 3 jul. 2020.

BARROS-DELBEN, P. et al. SAÚDE MENTAL EM SITUAÇÃO DE EMERGÊNCIA: COVID-19. Debates em Psiquiatria, v. 1, n. 2, p. 18-28, 2020. Disponível em: https://d494f813-3c95-463a-898cea1519530871.filesusr.com/ugd/c37608_6bd285d2f02b40098a94c81c49d 603b8.pdf. Acesso em: 6 jul. 2020.

BIRON, M. et al. Structuring for innovative responses to human resource challenges: A skunk works approach. Human Resource Management Review, v. 30, n. 3, p. 100768, 2020. Disponível em: http://www.sciencedirect.com/science/article/pii/S1053482220300413. Acesso em: 3 jul. 2020.

BROOKS, S. K. et al. The psychological impact of quarantine and how to reduce it: rapid review of the evidence. The Lancet, v. 395, n. 10227, p. 912920, 2020. Disponível em: https://doi.org/10.1016/S0140-6736(20)304608. Acesso em: 25 ago. 2020.

CDC COVID-19 RESPONSE TEAM. Severe Outcomes Among Patients with Coronavirus Disease 2019 (COVID-19) - United States, February 12March 16, 2020. MMWR Morb Mortal Wkly Rep, v. 69, n. 12, p. 343-346, 2020. Disponível em: https://pubmed.ncbi.nlm.nih.gov/32214079/. Acesso em: 3 jul. 2020.

CRUZ, R. M. et al. COVID-19: emergência e impactos na saúde e no trabalho. Revista Psicologia Organizaçôes e Trabalho, v. 20, p. I-III, 2020. Disponível em: http://pepsic.bvsalud.org/scielo.php?script=sci_arttext\&pid=S198466572020000200001\&nrm=iso. Acesso em: 4 jul. 2020.

FARO, A. et al. COVID-19 e saúde mental: a emergência do cuidado. Estudos de Psicologia (Campinas), v. 37, 2020. Disponível em: http://www.scielo.br/scielo.php?script=sci_arttext\&pid=S0103166X2020000100507\&nrm=iso. Acesso em: 3 ago. 2020.

GUILlAND, R.; CRUZ, R. M.; KASZUBOWSKI, E. Propriedades Psicométricas do Inventário de Fatores Psicológicos de Doenças Relacionadas ao Trabalho: Um Estudo com Trabalhadores de Frigoríficos. Psico-USF, v. 
23

p.

539-554,

2018.

Disponível

em:

http://www.scielo.br/scielo.php?script=sci_arttext\&pid=S1413-

82712018000300539\&nrm=iso. Acesso em: 4 fev. 2020.

IIVARI, N.; SHARMA, S.; VENTÄ-OLKKONEN, L. Digital transformation of everyday life - How COVID-19 pandemic transformed the basic education of the young generation and why information management research should care? International Journal of Information Management, v. 52, p. 102183, 2020. Disponível em: http://www.sciencedirect.com/science/article/pii/S0268401220310264. Acesso em: 13 ago. 2020.

LANA, R. M. et al. Emergência do novo coronavírus (SARS-CoV-2) e o papel de uma vigilância nacional em saúde oportuna e efetiva. Cadernos de Saúde Pública, v. 36, 2020. Disponível em: http://www.scielo.br/scielo.php?script=sci_arttext\&pid=S0102311X2020000300301\&nrm=iso. Acesso em: 6 ago. 2020.

LEE, S. A. Coronavirus Anxiety Scale: A brief mental health screener for COVID-19 related anxiety. Death Studies, v. 44, n. 7, p. 393-401, 2020. Disponível em: https://pubmed.ncbi.nlm.nih.gov/32299304. Acesso em: 7 ago. 2020.

LEI, L. et al. Comparison of Prevalence and Associated Factors of Anxiety and Depression Among People Affected by versus People Unaffected by Quarantine During the COVID-19 Epidemic in Southwestern China. Medical science monitor: international medical journal of experimental and clinical research, v. 26, p. e924609-e924609, 2020. Disponível em: https://pubmed.ncbi.nlm.nih.gov/32335579

https://www.ncbi.nlm.nih.gov/pmc/articles/PMC7199435. Acesso em: 1 jul. 2020 .

LIANG, L. et al. The Effect of COVID-19 on Youth Mental Health. The Psychiatric Quarterly, v. 91, n. 3, p. 841-852, 2020. Disponível em: https://pubmed.ncbi.nlm.nih.gov/32319041. Acesso em: 10 ago. 2020.

LIU, N. et al. Prevalence and predictors of PTSS during COVID-19 outbreak in China hardest-hit areas: Gender differences matter. Psychiatry Research, v. 287, p. 112921, 2020. Disponível em: 
http://www.sciencedirect.com/science/article/pii/S016517812030545X. Acesso em 12 ago. 2020.

LU, W. et al. Psychological status of medical workforce during the COVID19 pandemic: A cross-sectional study. Psychiatry research, v. 288, p. 112936 , 2020. Disponível em: https://pubmed.ncbi.nlm.nih.gov/32276196. Acesso em: 4 ago. 2020.

MARTINS, B. G. et al. Escala de Depressão, Ansiedade e Estresse: propriedades psicométricas e prevalência das afetividades. Jornal Brasileiro de Psiquiatria, v. 68, p. 32-41, 2019. Disponível em: http:/www.scielo.br/scielo.php?script=sci_arttext\&pid=S004720852019000100032\&nrm=iso. Acesso em: 5 mai. 2020.

MELLO, L. D.; TER-MINASSIAN, T. Digitalisation challenges and opportunities for subnational governments. OECD iLibrary, n. 31, 2020. Disponível em: https://www.oecd-ilibrary.org/content/paper/9582594a-en. Acesso em: 7 ago. 2020.

NORTON, P. J. Depression Anxiety and Stress Scales (DASS-21): psychometric analysis across four racial groups. Anxiety Stress Coping, v. 20, n. 3, p. 253-65, 2007. Disponível em: https://pubmed.ncbi.nlm.nih.gov/17999228. Acesso em: 2 fev. 2020.

QIU, J. et al. A nationwide survey of psychological distress among Chinese people in the COVID-19 epidemic: implications and policy recommendations. General Psychiatry, v. 33, n. 2, p. e100213, 2020. Disponível em: https://pubmed.ncbi.nlm.nih.gov/32215365. Acesso em: 12 ago. 2020.

RANGANATHAN, P.; AGGARWAL, R. Study designs: Part 1 - An overview and classification. Perspectives in clinical research, v. 9, n. 4, p. 184186, 2018. Disponível em: https://pubmed.ncbi.nlm.nih.gov/30319950. Acesso em: 12 dez. 2020.

https://www.ncbi.nlm.nih.gov/pmc/articles/PMC6176693/. Acesso em: 4 dez. 2019.

SCHMIDT, B. et al. Saúde mental e intervençôes psicológicas diante da pandemia do novo coronavírus (COVID-19). Estudos de Psicologia 
(Campinas),

v. $\quad 37$,

2020.

Disponível

em:

http://www.scielo.br/scielo.php?script=sci_arttext\&pid=S0103166X2020000100501\&nrm=iso. Acesso em: 28 jul. 2020.

SCHWARTZ, J.; KING, C. C.; YEN, M. Y. Protecting Healthcare Workers During the Coronavirus Disease 2019 (COVID-19) Outbreak: Lessons From Taiwan's Severe Acute Respiratory Syndrome Response. Clinical infectious diseases : an official publication of the Infectious Diseases Society of America, v. 71, n. 15, p. 858-860, 2020. Disponível em: https://pubmed.ncbi.nlm.nih.gov/32166318. Acesso em: 12 jul. 2020.

SHACHAM, M. et al. COVID-19 Factors and Psychological Factors Associated with Elevated Psychological Distress among Dentists and Dental Hygienists in Israel. International journal of environmental research and public health, v. 17, n. 8, 2020. Disponível em: https://pubmed.ncbi.nlm.nih.gov/32331401. Acesso em: 13 ago. 2020.

SHIGEMURA, J. et al. Public responses to the novel 2019 coronavirus (2019-nCoV) in Japan: Mental health consequences and target populations. Psychiatry Clin Neurosci, v. 74, n. 4, p. 281-282, 2020. Disponível em: https://pubmed.ncbi.nlm.nih.gov/32034840. Acesso em: 19 jul. 2020.

SOMINSKY, L.; WALKER, D. W.; SPENCER, S. J. One size does not fit all - Patterns of vulnerability and resilience in the COVID-19 pandemic and why heterogeneity of disease matters. Brain, behavior, and immunity, v. 87, p. 1-3, 2020. Disponível em: https://pubmed.ncbi.nlm.nih.gov/32205119. Acesso em: 24 jul. 2020.

TAN, W. et al. Is returning to work during the COVID-19 pandemic stressful? A study on immediate mental health status and psychoneuroimmunity prevention measures of Chinese workforce. Brain, Behavior, and Immunity, v. 87, p. 84-92, 2020. Disponível em: http://www.sciencedirect.com/science/article/pii/S0889159120306036. Acesso em: 8 ago. 2020.

TEMSAH, M. H. et al. The psychological impact of COVID-19 pandemic on health care workers in a MERS-CoV endemic country. Journal of Infection and Public Health, v. 13, n. 6, p. 877-882, 2020. Disponível em: 
http://www.sciencedirect.com/science/article/pii/S1876034120304871. Acesso em: 8 jun. 2020.

THE LANCET. COVID-19: protecting health-care workers. The Lancet, v. 395, n. 10228, p. 922, 2020. Disponível em: https://doi.org/10.1016/S01406736(20)30644-9. Acesso em: 25 ago. 2020.

TÓFOLI, L. F.; ANDRADE, L. H.; FORTES, S. Somatização na América Latina: uma revisão sobre a classificação de transtornos somatoformes, síndromes funcionais e sintomas sem explicação médica. Brazilian Journal of Psychiatry, v. 33, p. s59-s69, 2011. Disponível em: http:/www.scielo.br/scielo.php?script=sci_arttext\&pid=S1516$44462011000500006 \&$ nrm=iso. Acesso em: 12 dez. 2019.

UNESCO. Education: From disruption to recovery 2020. Disponível em: https://en.unesco.org/covid19/educationresponse. Acesso em: 24 ago. 2020.

USHER, K.; DURKIN, J.; BHULLAR, N. The COVID-19 pandemic and mental health impacts. International Journal of Mental Health Nursing, v. 29, n. 3, p. 315-318, 2020. Disponível em: https://onlinelibrary.wiley.com/doi/abs/10.1111/inm.12726. Acesso em: 9 mai. 2020.

WANG, Y. et al. Study on the public psychological states and its related factors during the outbreak of coronavirus disease 2019 (COVID-19) in some regions of China. Psychology, health \& medicine, p. 1-10, 2020. Disponível em: https://pubmed.ncbi.nlm.nih.gov/32223317. Acesso em: 15 jun. 2020.

WORLD HEALTH ORGANIZATION. Mental health and psychosocial considerations during the COVID-19 outbreak, 18 March 2020. World Health Organization, 2020 Disponível em: https://apps.who.int/iris/handle/10665/331490. Acesso em: 1 ago. 2020.

ZHANG, S. X. et al. Unprecedented disruption of lives and work: Health, distress and life satisfaction of working adults in China one month into the COVID-19 outbreak. Psychiatry Research, v. 288, p. 112958, 2020. Disponível em: http://www.sciencedirect.com/science/article/pii/S0165178120306521. Acesso em: 1 ago. 2020. 
344 Polyphonía, v. 31/1, jan.-jun. 2020

Recebido em: 01 set. 2020

Aceito em: 20 set. 2020 